Research Article

\title{
Determining the Degree of Characteristics for Internet of Healthcare Devices Using Fuzzy ANP
}

\author{
Lin Wen \\ Department of Medical Equipment, Sichuan Provincial People's Hospital, \\ University of Electronic Science and Technology of China, Chengdu, China \\ Correspondence should be addressed to Lin Wen; linwen044@gmail.com
}

Received 30 April 2021; Accepted 9 June 2021; Published 29 June 2021

Academic Editor: Shah Nazir

Copyright (c) 2021 Lin Wen. This is an open access article distributed under the Creative Commons Attribution License, which permits unrestricted use, distribution, and reproduction in any medium, provided the original work is properly cited.

With the revolution in Internet and digital technology, every organization is adopting digital things to carry out their day-to-day activities. Internet of Things (IoT) is a concept that is used to connect various devices over the Internet to increase the production and quality of service, deliver a huge amount of data in seconds, and automate the processes. IoT implementation in the health sector has changed the typical setup into smart and intelligent setup. With the "smart" and "Intelligent" abilities of IoT devices such as sensors and with the collaboration of humans and computers, physical processes can be monitored and, based on the received data, optimal decisions can ultimately be taken. IoT applications in healthcare will increase flexibility, patient's care, quality of health, and control of diseases. As the IoT combines various heterogeneous devices and the inappropriate determination of the degree of characteristics for Internet of healthcare $(\mathrm{IoH})$ devices may affect the efficiency of services, this research is carried out with the use of decision support system and application of fuzzy analytic network process (fuzzy ANP) was used for optimal determination of the degree of characteristics for IoH devices based on unique properties which would greatly increase the efficiency of industry. The experimental results are efficient and show the usefulness of the approach.

\section{Introduction}

The evolution of information technology to the IoT has demonstrated that a highly intelligent ecosystem can be built by connecting an information network autonomously via routers from OS built gadgets and computers [1]. However, there are some security issues with IoT, such as understanding the transition from offline to online infrastructure, handling temporal aspects of security, resolving the implementation gap for best practice, and dealing with development complexity [2]. Industrial processes have been turned into completely digitized smart processes as a result of Industry 4.0, which involves IoT, Industrial Internet, smart manufacturing, and cloud-based manufacturing and is still a futuristic yet practical idea. It has placed strict human constraints in the manufacturing process in order to achieve quality improvement and an emphasis on valueadded activities while eliminating waste [3]. Due to heterogeneous devices, multiple channel impairments, attenuation, spontaneous variations, and shadowing impacts occur in industrial scenarios where devices interact dynamically and in a stable and efficient manner with one another using wireless technology [4]. Flexibility, mass customization, service efficiency, and productivity are all growing as a result of Industry 4.0 [5]. To control physical processes and make intelligent choices, modern industrial production systems depend on real-time cooperation and consultation with humans, machines, sensors, and other systems. Resources are transformed into intelligent objects so that they can feel, function, and behave in a smart world, which is an important part of Industry 4.0 [6]. Industrial Internet of Things (IIoT) has revolutionized the digital industry by allowing for product accountability, integrity control, and delivery cost [7].

IoT solutions lead to the formation of innovative and efficient innovations in today's manufacturing environments, with the aim of increasing operational efficiency in growing fast responding factories [8]. Flexible 
manufacturing systems allow all devices to communicate via the Internet of Things (IIoT). Automotive automakers' willingness to adapt processes to achieve ultimate versatility would help them maintain efficiency in an increasingly global marketplace [9]. During the production process, numerous devices capture signal and upload it to a cloud server via the Internet. Since a cloud server cannot be fully trusted, data authenticity should be maintained before data is outsourced to the cloud server, ensuring that only approved users or devices have access to the authentic data from various topography areas [10]. The IoT combines various heterogeneous devices that may produce certain risks to data such as data loss, decreased throughput, time delays, latency problem, and security and privacy issues.

Various research studies have been carried out on IoT and IIoT which addressed different areas of research including smart communication, intelligent factories, and Industry 4.0. Research works have been done in the area and some researchers suggested approaches for enhancing the efficiency and effectiveness of industry with IoT applications. However, no study revealed the problem of determining the degree of characteristics of IoH devices in the IoT setup. To overcome this issue, the proposed research is carried out as a decision support system and the applications of fuzzy ANP were used for optimum determination of the degree of characteristics for IoH devices based on exclusive properties which would significantly increase the efficiency of industry.

The remainder of this paper is organized as follows: work related to the area of the research is given in Section 2. The methodology of the paper with subheadings is briefly described in Section 3. Section 4 discusses the results and discussion of the paper. The conclusion of the paper is presented in Section 5.

\section{Related Work}

Researchers are trying to come across approaches, tools, and solutions for effective IoT in industry. Various approaches have been devised. The asynchronous altering direction method of multipliers algorithm was used to solve the global consensus cross-layer optimization problem that is associated with the control of industrial health status [11]. For observing the issue of a single architecture that complies with Industry 4.0 standards as they apply to businesses, the research provided a fundamental idea for free, safe, stable, near real-time, standardised communication interfaces [12]. DDoS mitigation employs the fog computing philosophy. Real-time traffic filtering through field firewall tools is used in the mitigation process, and data from decentralized dedicated servers is correlated for accurate decision-making [13]. In order to break the deadlock in Industry 4.0, a job shop scheduling method is adopted, and a new assessment feature is created, using the Asset Administrative Shell (AAS) [14]. Logistics 4.0 is the result of the IIoT revolutionizing the logistic domains by offering accountability (supply chain visibility), integrity management, and commodity delivery cost. In the scope of Logistics 4.0, some insights on the necessary criteria and concerns for organizations to be effective and fully operational are highlighted
[7]. Flexibility, mass customization, consistency, and efficiency are all gaining ground as a result of Industry 4.0. The importance of maintenance in Industry 4.0, as well as the potential for new quality control and production development, is highlighted. Present maintenance measures are trending toward "remote maintenance" and the appealing prospect of "self-maintenance." It has been highlighted that the human element is also a significant factor in achieving various goals such as flexibility and efficiency [5].

Several channel impairments, attenuation, random fluctuations, and shadowing impacts, as well as non-line-ofsight, occur in industrial scenarios where devices interact freely and in a stable and efficient manner with one another using wireless technology. For channel model evaluation, performance estimation techniques such as acquired signal power, packet loss likelihood, RMS (Root Mean Square) error, and RMS delay spread are examined. This research may be used to satisfy the requirements for adequate IIoT channel modelling [4]. Industry 4.0 places a high value on industrial automation. Typical tools are transformed into intelligent objects capable of sensing, acting, and behaving in a smart world. Smart factory and cloud manufacturing are all terms used to describe different types of smart manufacturing systems. Intelligent production is enabled by cyber-physical systems, cloud computing, big data analytics, and information and communications technology (ICT) [6]. The NICE Nanogrid was used to build laboratory-based IIoT-driven software and controls framework for the Shenhua community as part of a NICE smart device initiative. The aim is to create an open architecture-based Industrial Smart Energy Consortium to bring together industrial partners, academic institutions, module suppliers, equipment vendors, and other interested parties to investigate and apply to a test-bed-centric open laboratory template and platform for next-generation energy-oriented smart industry applications [1]. The rapid hardware proliferation of a complicated smart sensor communication system was accomplished using a high-level system design. The GRAFCET is being used to design the system's structural actions as well as internal functional units. A sequential-concurrent hybrid discrete event scheme is used to describe the actions of every unit. For a smart grid sensor and an intelligent gateway module, high-level synthesis rules were used to generate VHSIC hardware description language- (VHDL-) target efficient hardware. Ultimately, to incorporate all smart system components into a complicated embedded system, these embedded systems controllers are generated automatically, and a hardware circuitry is synthesized [15].

The biogeography-based optimization and the Markov decision process are combined in a hybrid model. The MDP is used to determine the likelihood of selecting a radio access tool, which serves as an input to the $\mathrm{BBO}$ process. As a result, in a heterogeneous network, the BBO uses the described multipoint algorithm to decide the best RAT [16]. The security of the IoT layered architecture, as well as the standards used for communication, networking, and maintenance, was investigated. Security specifications for IoT were established, as well as current threats, risks, and state-of-the-art 
TABle 1: Existing approaches in the area.

\begin{tabular}{|c|c|c|}
\hline No. & Title & Year Ref. \\
\hline 1 & Maintenance Transformation through Industry 4.0 Technologies: A Systematic Literature Review & $2020[5]$ \\
\hline 2 & Attacks on the Industrial Internet of Things-Development of a Multilayer Taxonomy & 2020 [25] \\
\hline 3 & $\begin{array}{c}\text { Comparative Link-Level Analysis and Performance Estimation of Channel Models for IIoT (Industrial-IoT) Wireless } \\
\text { Communications }\end{array}$ & $2020[4]$ \\
\hline 4 & Research on the Optimization of IIoT Data Processing Latency & $2020[18]$ \\
\hline 5 & Deep Robust Cramer Shoup Delay Optimized Fully Homomorphic for IIOT Secured Transmission in Cloud Cc & $2020[10]$ \\
\hline 6 & Intelligent Equipment Design Assisted by Cognitive Internet of Things and Industrial Big Data & $2020[26]$ \\
\hline 7 & Big Data and Disruptive Computing Platforms Braced Internet of Things: Facets and Trends & $2020[27]$ \\
\hline 8 & Industrial Internet of Things Based Ransomware Detection Using Stacked Variational Neural Network & $2019[22]$ \\
\hline 9 & The Need for Deterministic Virtualization in $\mathrm{t}$ & 2019 [23] \\
\hline 10 & A Fog Computing Based Approach to DDoS Mit & $2019[13]$ \\
\hline 11 & lodel for Network Selection & $2019[16]$ \\
\hline 12 & Towards Distributed IoT/ & $2019[11]$ \\
\hline 13 & ing Systems Using Il & 2019 [9] \\
\hline 14 & Big & 2019 [28] \\
\hline 15 & An Efficient Intrusion I & 2019 [20] \\
\hline 16 & A Mic & 2018 [21] \\
\hline 17 & 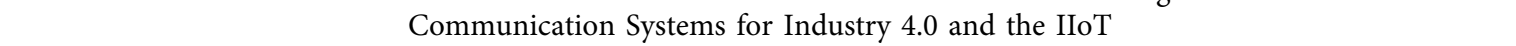 & 2018 [12] \\
\hline 18 & M & $2018[14]$ \\
\hline 19 & Enabling Industrial Internet of Thin & 2018 \\
\hline 20 & 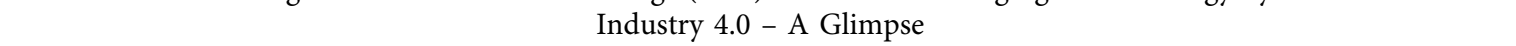 & 2018 \\
\hline 21 & $\begin{array}{c}\text { Parallel Computing and Network Analytics for Fast Industrial Internet-of-Things (IIoT) Machine Information } \\
\text { Processing and Condition Monitoring }\end{array}$ & 2018 \\
\hline 22 & Avoiding the Internet of Insecure In & 2018 \\
\hline 23 & IoT Secur & 2018 [17] \\
\hline 24 & $\mathrm{w}$ & 2017 [7] \\
\hline 25 & $\mathrm{e} \mathrm{Co}$ & 2017 \\
\hline 26 & High-Level Modeling a & 2017 [15] \\
\hline 27 & $\begin{array}{c}\text { rge-Scale Web QoS Prediction Scheme for the Industrial Internet of Things Based on a Kernel Machine Learning } \\
\text { Algorithm }\end{array}$ & 2016 \\
\hline 28 & Industrial Internet of Things Monitoring Solution for Advanced Predictive Maintenance Applications & $2016[8]$ \\
\hline
\end{tabular}

solutions. It was discovered that blockchain could be a major driver in resolving many IoT security issues [17]. To address high latency when processing industrial data on cloud, an Industrial Internet of Things cloud-fog hybrid network architecture is proposed. Framework uses edge devices like routers and switches to build a fog computing layer between the cloud server and the production equipment in the production equipment region. A distributed computing approach for multiple devices is proposed because the computing power of the edge devices in fog computing is very low. The constrained particle swarm optimization load balancing algorithm based on the simulated annealing approach is used to achieve the goal of minimizing task processing delays (SAPSO-LB) [18]. The kernel least mean square (KLMS) algorithm is used to suggest a data-driven scheme for predicting missing QoS values for the IIoT. For each known QoS entry, the Pearson correlation coefficient (PCC) is first applied during the data prediction process to find related QoS values from similar service users and web service objects. The secret relationships between all known QoS data and corresponding QoS data with the highest similarities are then investigated using KLMS [19]. The NGS-PlantOne system is a cutting-edge Industrial IoT (IIoT) solution that allows ubiquitous tracking of machinery and equipment via rechargeable IoT sensing devices, allowing for the creation of advanced planned maintenance applications in the IIoT scenario. The generated IIoT solution's reliability results are promising [8]. Flexible manufacturing systems (FMS) allow all systems to function via IIoT. The willingness of automakers to adapt processes in order to achieve ultimate versatility would help them maintain profitability in an increasingly global marketplace. The key elements to effective implementation of smart manufacturing for the automotive sector were investigated, as well as flexible manufacturing systems that use IIoT [9].

For privacy protection, Robust Cramer Shoup Delay Optimized Fully Homomorphic (RCS-DOFH) is proposed. To decode privately induced signals and their weighted variables, the RCSD protocol is used. The encoding is then carried out using DOFHE method. After that, the encoding territory's activation role is calculated [10]. A software architecture is proposed which incorporates concepts from the IoT environment, various industrial automation domains, and modern IT and cloud architectures. Application patterns are used to gain a better understanding of the problem domain and to create a consistent software architecture throughout the development of the software architecture. The software architecture's resulting lightweight and modular nature, combined with support for modern development approaches (containerization, CI, CD), makes it ideal for deployment on cloud, fog, and edge devices in industrial and nonindustrial IoT environments [20]. In the industrial 
automation market, the Internet of Things (IoT) and cyberphysical system (CPS) principles have been introduced. Due to tighter dependability and real-time constraints, the IoT concept cannot be completely adopted in IIoT environments [21]. A detection model based on a fully connected neural network and a stacked Variational Autoencoder (VAE) was proposed which can learn the latent structure of device activities and reveal ransomware actions [22]. Real-time virtualization is widely acknowledged as a primary enabler of fog computing and the IIoT. There are some requirements that any hypervisor that claims to be a deterministic virtualization solution for IIoT must meet. Current studies on real-time virtualization were examined to explain the tradeoff between versatility and deterministic execution. Hypervisors that fulfil all of the criteria for deterministic virtualization have been defined as a requirement [23].

\section{Methodology}

The following sections briefly show the methodology of the paper.

3.1. Existing Approaches in the Area. Industry 4.0, which includes Internet of Things, Industrial Internet, Smart Manufacturing, and Cloud-based Production, is still a futuristic yet practical term. Industry 4.0 refers to the strict incorporation of humans into the production process in order to achieve quality improvement, an emphasis on value-added operations, and the avoidance of waste. An overview of Industry 4.0 was provided, as well as an understanding of the nine pillars of Industry 4.0 and their implementations, in addition to identifying the problems and issues that arise when implementing Industry 4.0 [3]. A new approach to large-scale IIoT system information processing, network modelling, fault detection, and fault diagnosis has been suggested. A dynamic warping algorithm was first implemented to describe the distinction of system signatures (e.g., power profiles during operations). Second, a stochastic network embedding algorithm was created for constructing a large-scale network of IIoT machines, with the dissimilarity between machine signatures maintained in the network node-to-node distance. A parallel computing method was designed for effective system modelling of largescale IIoT-enabled devices, using the power of multiple processors. According to experimental results, the proposed technique efficiently and effectively characterizes signature differences in both cycle-to-cycle and machine-to-machine dimensions. [24]. A multilayer taxonomy was used to distinguish similarities and differences between attacks on the IIoT by incorporating IT protection, IIoT, and risk mitigation into a multidisciplinary approach. The study also encourages organizations and their value-creation networks to identify, record, and share events [25]. Admiring the transition from offline to online infrastructure, handling temporal aspects of protection, resolving the identified gaps for best practice, and dealing with infrastructural complexity are all security issues posed by IoT. Noncompliance risks can arise as a result of smart energy systems. The study focuses on
TABle 2: Characteristics and features of IoH devices.

\begin{tabular}{lc}
\hline Characteristics of IoH devices & References \\
\hline Efficiency & {$[29-34]$} \\
Reliability & {$[29,33,35,36]$} \\
Security & {$[29-31,36-38]$} \\
Privacy & {$[29,35,37,39]$} \\
Real time & {$[29,30,40]$} \\
Intelligent & {$[30,32,34,35,41]$} \\
High sensing & {$[30,32,36]$} \\
Availability & {$[29,31,41]$} \\
Interoperability & {$[29,42]$} \\
\hline
\end{tabular}

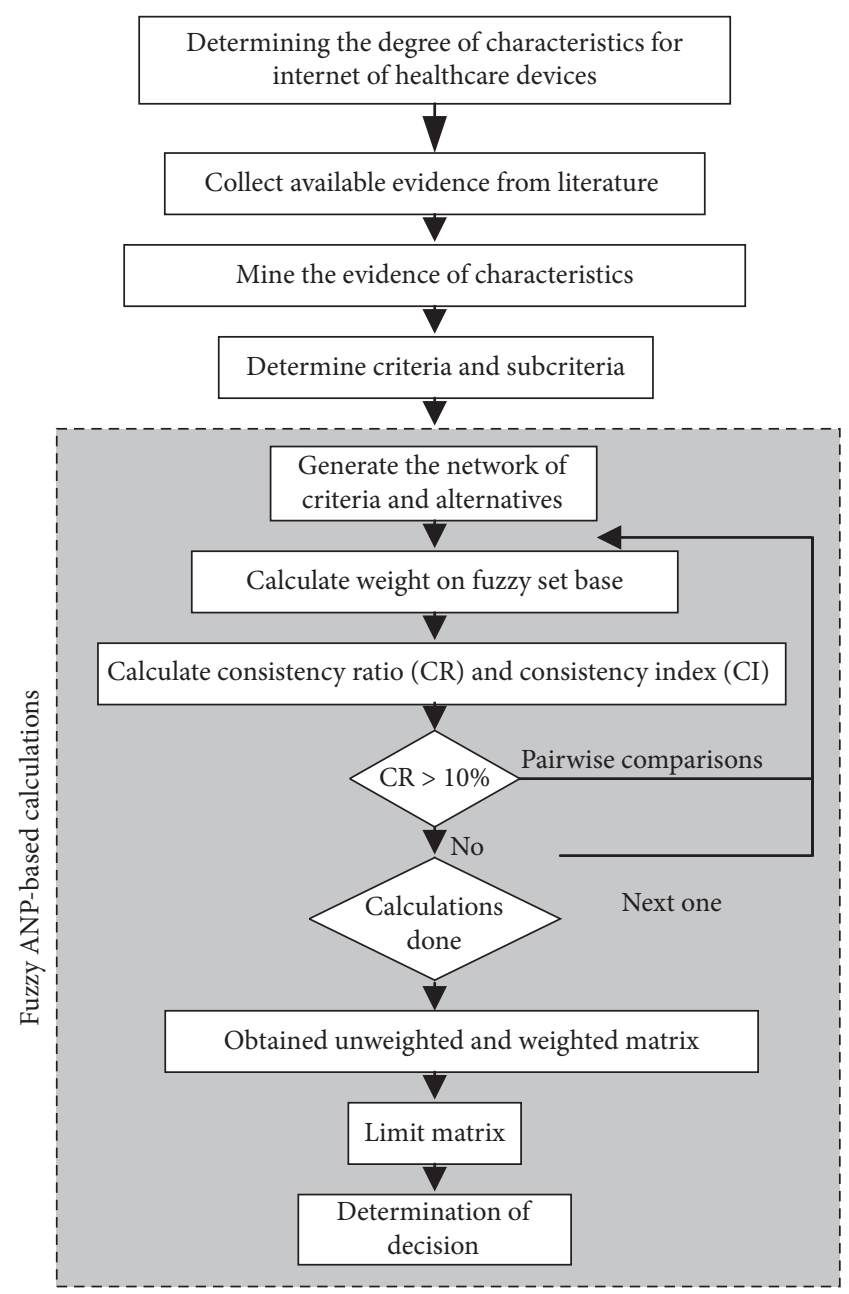

FIgURE 1: Process of the proposed research for determination of characteristics of IoH devices.

how to escape these dangers [2]. Table 1 shows some of the existing approaches proposed by the researchers in the field.

3.2. Defining the Characteristics of IoH Devices. The existing literature in the area of research was studied for identifying various features and characteristics of the IoH for determination of degree of characteristics of devices. More features were identified, but these features' analysis was quite tricky, so the features were then reduced and the significant features were 


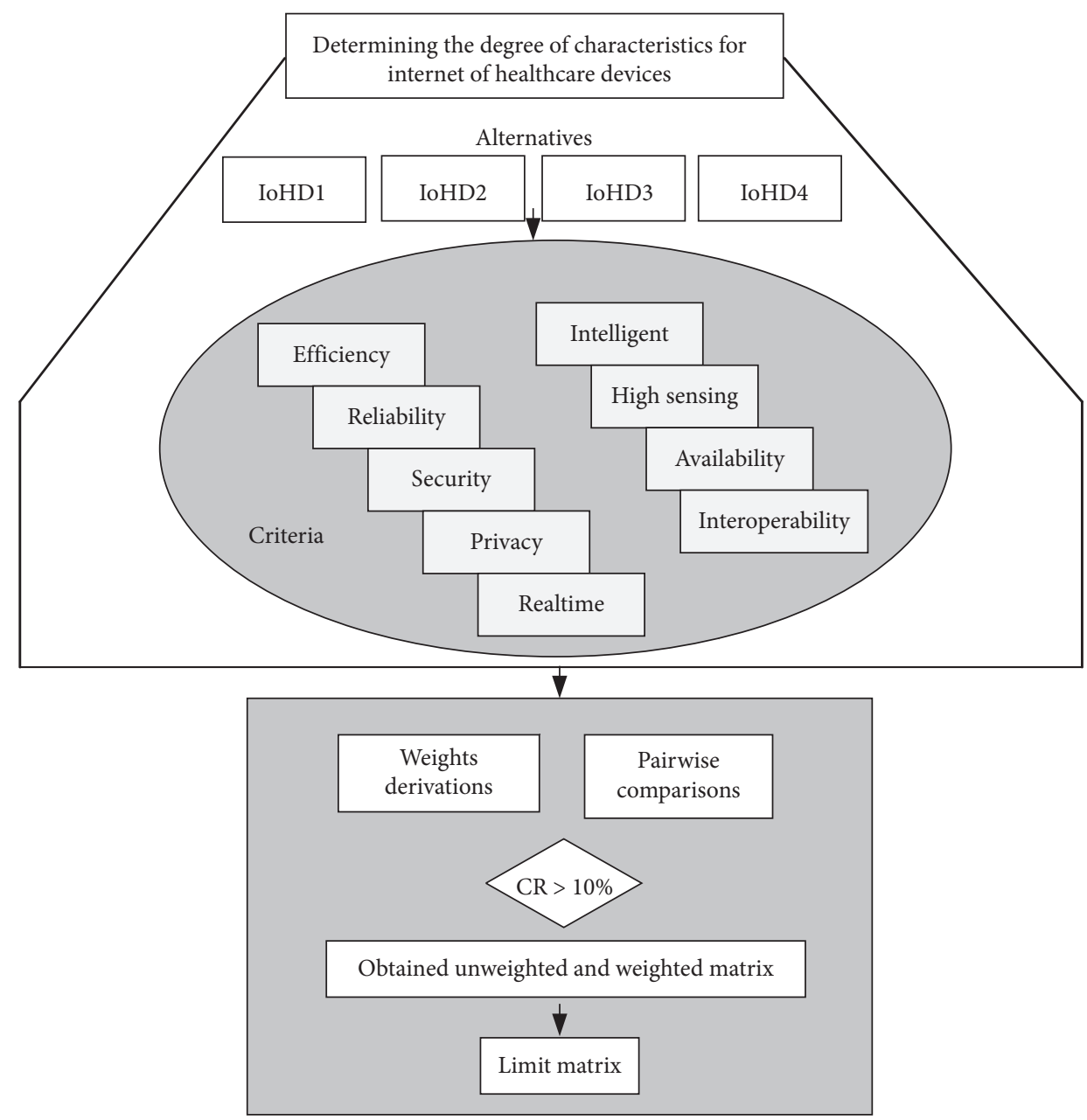

Figure 2: Network of the criteria, alternatives, and the application of fuzzy ANP.

selected. Table 2 shows the common features and characteristics of the $\mathrm{IoH}$ devices along with the references given.

\subsection{Process of the Fuzzy ANP for Determining the Charac-} teristics of IoH Devices. Fuzzy ANP is the combination of fuzzy and ANP which are used for uncertain, vague, and complex situation where decision-making is difficult. The proposed method has used the network of elements and clusters and fuzzy set of membership range from $1^{\prime}$ to $9^{\prime}$ domain. Fuzzy set is described by membership function [43]. It is extremely beneficial where decision-making is about uncertainty and vagueness. Here the decision can be made in linguistics variable instead of numbers; on the other side, the ANP is a more general form of analytic hierarchy process. In fuzzy ANP, triangular membership functions are used to represent opinions and the triangular membership function has $(l, m, u)$ parameters which are defined as $1 \leq m \leq u$. In the proposed method, triangular fuzzy numbers from $1^{\prime}$ to $9^{\prime}$ are used for pairwise comparisons of the selection process.
Figure 1 depicts the proposed research process for determination of characteristics of IoH devices.

Figure 2 briefly shows the network of the criteria, alternatives, and the application of fuzzy ANP. Four different alternatives were presented for the process of research and nine criteria were presented. The whole process of the experiments was followed for conducting the proposed process.

Once the criteria, alternatives, and their network of elements are drawn, the process of fuzzy ANP was started and various comparison measures have been done. Table 3 gives the representation of values assigned to the Internet of healthcare device (IoHD) 1.

After assignment of the values to different features of the IoHD, the process of comparisons was started. Table 4 depicts the value obtained of Table 3 after comparison process.

The same process was applied on the remaining IoHD. The comparisons process for each feature was done. Table 5 describes the value assignment to the available features of IoHD. 
TABle 3: Value assignment to IoHD1.

\begin{tabular}{lccccccccc}
\hline & Efficiency & Reliability & Security & Privacy & Real time & Intelligent & High sensing & Availability & Interoperability \\
\hline Efficiency & 1 & {$[2,4]$} & {$[2,4]$} & {$[8,10]$} & {$[3,5]$} & {$[4,6]$} & {$[6,8]$} & {$[8,10]$} & {$[8,10]$} \\
Reliability & {$[1 / 2,1 / 4]$} & 1 & {$[1,3]$} & {$[2,4]$} & {$[1,3]$} & {$[2,4]$} & {$[4,6]$} & {$[2,4]$} & {$[8,10]$} \\
Security & {$[1 / 2,1 / 4]$} & {$[1,1 / 3]$} & 1 & {$[1,3]$} & {$[1,3]$} & {$[1,3]$} & {$[1,3]$} & {$[2,4]$} & {$[6,8]$} \\
Privacy & {$[1 / 8,1 / 10]$} & {$[1 / 2,1 / 4]$} & {$[1,1 / 3]$} & 1 & {$[1,3]$} & {$[2,4]$} & {$[1,3]$} & {$[1,3]$} & {$[4,6]$} \\
Real time & {$[1 / 3,1 / 5]$} & {$[1,1 / 3]$} & {$[1,1 / 3]$} & {$[1,1 / 3]$} & 1 & {$[1,3]$} & {$[1,3]$} & {$[1,3]$} & {$[2,4]$} \\
Intelligent & {$[1 / 4,1 / 6]$} & {$[1 / 2,1 / 4]$} & {$[1,1 / 3]$} & {$[1 / 2,1 / 4]$} & {$[1,1 / 3]$} & 1 & {$[3,5]$} & {$[2,4]$} & {$[1,3]$} \\
High sensing & {$[1 / 6,1 / 8]$} & {$[1 / 4,1 / 6]$} & {$[1,1 / 3]$} & {$[1,1 / 3]$} & {$[1,1 / 3]$} & {$[1 / 3,1 / 5]$} & 1 & {$[4,6]$} & {$[1,3]$} \\
Availability & {$[1 / 8,1 / 10]$} & {$[1 / 2,1 / 4]$} & {$[1 / 2,1 / 4]$} & {$[1,1 / 3]$} & {$[1,1 / 3]$} & {$[1 / 2,1 / 4]$} & {$[1 / 4,1 / 6]$} & 1 & {$[1,3]$} \\
Interoperability & {$[1 / 8,1 / 10]$} & {$[1 / 8,1 / 10]$} & {$[1 / 6,1 / 8]$} & {$[1 / 4,1 / 6]$} & {$[1 / 2,1 / 4]$} & {$[1,1 / 3]$} & {$[1,1 / 3]$} & {$[1,1 / 3]$} & 1 \\
\hline
\end{tabular}

TABLE 4: Comparison process of Table 3.

\begin{tabular}{|c|c|c|c|c|c|c|c|c|c|c|}
\hline & Efficiency & Reliability & Security & Privacy & Real time & Intelligent & High sensing & Availability & Interoperability & EV \\
\hline Efficiency & 0.386 & 0.475 & 0.354 & 0.528 & 0.312 & 0.293 & 0.295 & 0.316 & 0.225 & 0.354 \\
\hline Reliability & 0.129 & 0.158 & 0.236 & 0.176 & 0.156 & 0.176 & 0.211 & 0.105 & 0.225 & 0.175 \\
\hline Security & 0.129 & 0.079 & 0.118 & 0.117 & 0.156 & 0.117 & 0.084 & 0.105 & 0.175 & 0.120 \\
\hline Privacy & 0.043 & 0.053 & 0.059 & 0.059 & 0.156 & 0.176 & 0.084 & 0.070 & 0.125 & 0.092 \\
\hline Real time & 0.096 & 0.079 & 0.059 & 0.029 & 0.078 & 0.117 & 0.084 & 0.070 & 0.075 & 0.077 \\
\hline Intelligent & 0.077 & 0.053 & 0.059 & 0.020 & 0.039 & 0.059 & 0.169 & 0.105 & 0.050 & 0.070 \\
\hline High sensing & 0.055 & 0.032 & 0.059 & 0.029 & 0.039 & 0.015 & 0.042 & 0.175 & 0.050 & 0.055 \\
\hline Availability & 0.043 & 0.053 & 0.039 & 0.029 & 0.039 & 0.020 & 0.008 & 0.035 & 0.050 & 0.035 \\
\hline Interoperability & 0.043 & 0.018 & 0.017 & 0.012 & 0.026 & 0.029 & 0.021 & 0.018 & 0.025 & 0.023 \\
\hline
\end{tabular}

TABle 5: Values assignment to the features.

\begin{tabular}{lcccc}
\hline & IoHD1 & IoHD2 & IoHD3 & IoHD4 \\
\hline IoHD1 & 1 & {$[4,6]$} & {$[3,5]$} & {$[1,3]$} \\
IoHD2 & {$[1 / 4,1 / 6]$} & 1 & {$[1 / 3,1 / 5]$} & {$[1 / 5,1 / 7]$} \\
IoHD3 & {$[1 / 3,1 / 5]$} & {$[3,5]$} & 1 & {$[1 / 2,1 / 4]$} \\
IoHD4 & {$[1,1 / 3]$} & {$[5,7]$} & {$[2,4]$} & 1 \\
\hline
\end{tabular}

TABle 6: Comparisons of Table 5.

\begin{tabular}{lcccrrr}
\hline & IoHD1 & IoHD2 & IoHD3 & IoHD4 & 0.571 & 0.470 \\
\hline IoHD1 & 0.513 & 0.313 & 0.485 & 0.030 & 0.061 \\
IoHD2 & 0.103 & 0.063 & 0.121 & 0.095 & 0.149 \\
IoHD3 & 0.128 & 0.250 & 0.364 & 0.286 & 0.320 \\
IoHD4 & 0.256 & 0.375 & & & \\
\hline
\end{tabular}

The same process of comparisons was done and the calculated values are obtained in Table 6.

\section{Results and Discussion}

The process of fuzzy ANP was done in order to achieve efficient results of the available IoHD for their effective usage and to determine the characteristics of the IoH devices. Initially, all the steps of the process were followed. After the comparisons process of all the features and the available IoHD, the values were combined into a single table called the unweighted matrix. Table 7 depicts the unweighted super matrix.
The unweighted super matrix was converted into the weighted super matrix. The purpose of this matrix was to obtain the limit matrix. Table 8 shows the weighted super matrix.

Table 8 was converted into limit matrix for the purpose of decision. This matrix was obtained through taking the power of the given matrix. The limit matrix is shown in Table 9. This matrix was achieved for the purpose of decision-making.

On the basis of the limit matrix obtained, the decisions regarding the available IoHDs are made. Figure 3 briefly represents the details of ranking of the available IoHDs. 


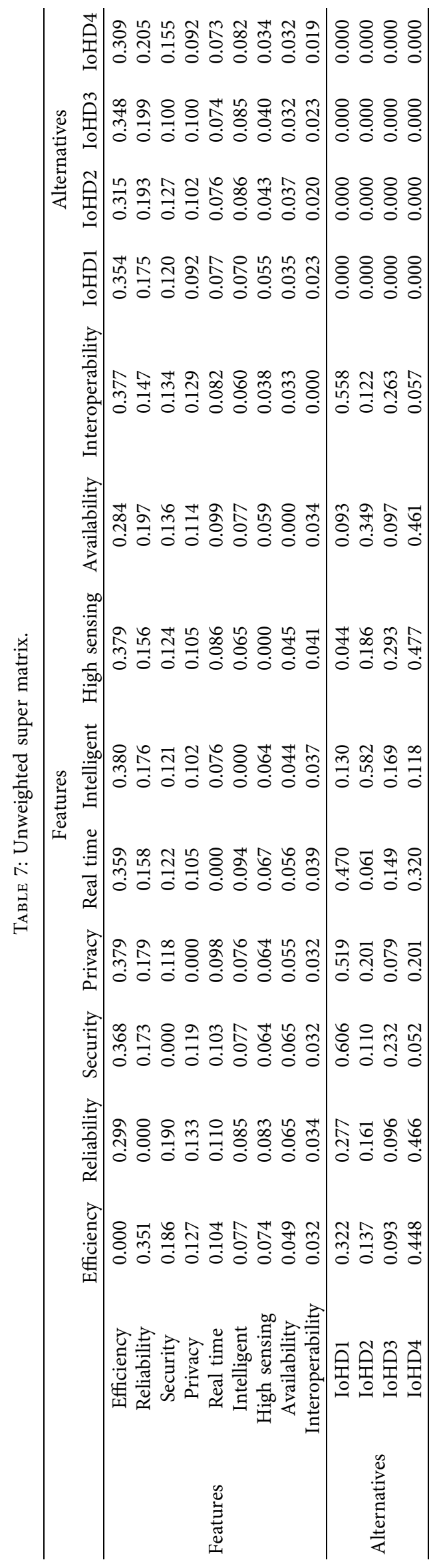




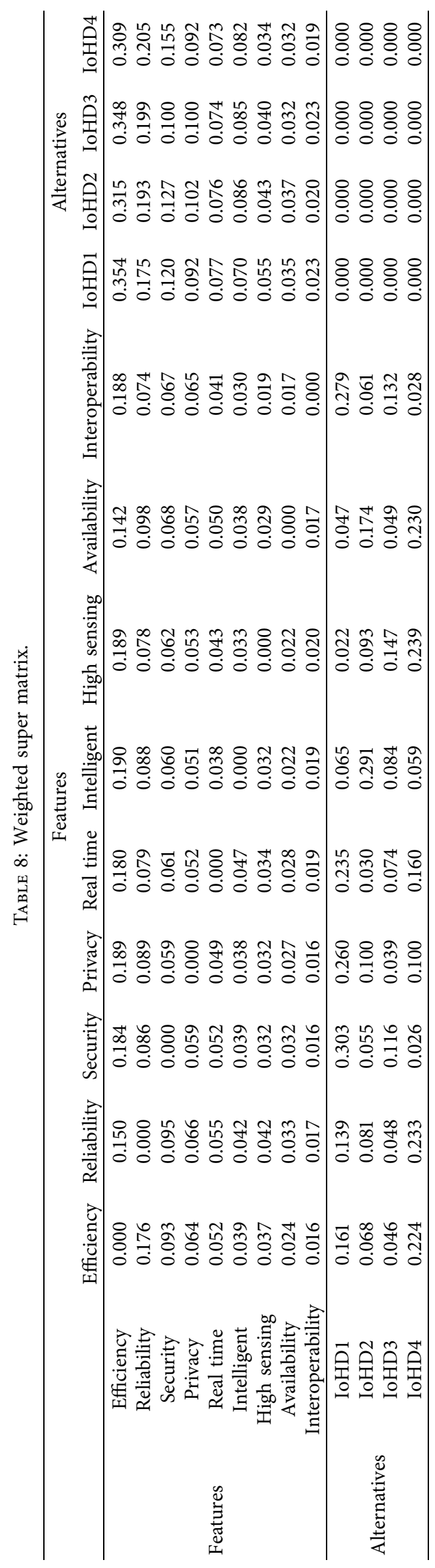




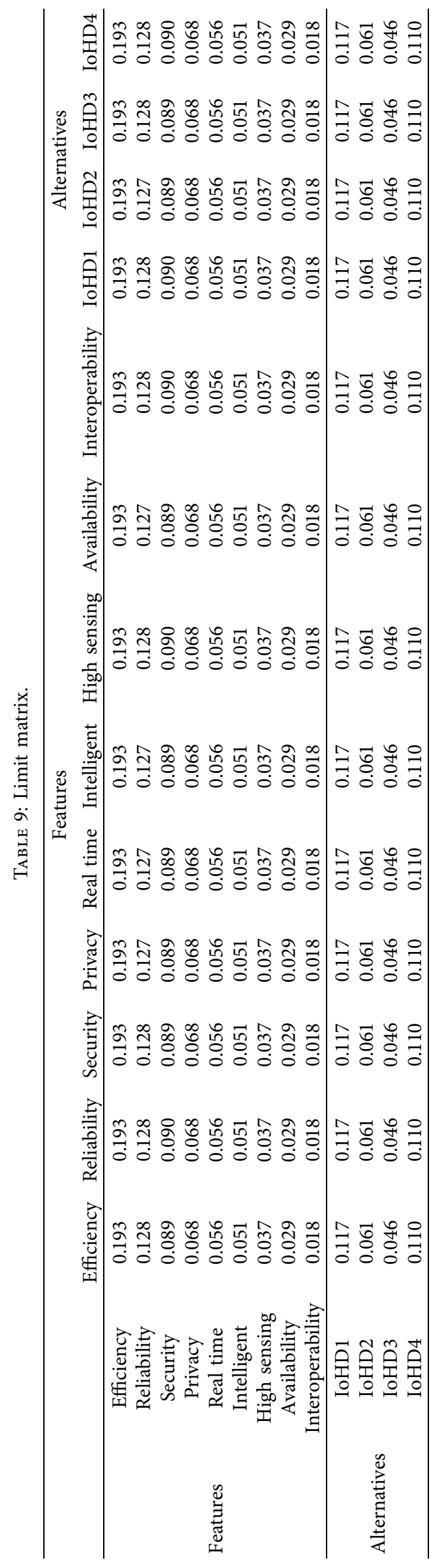




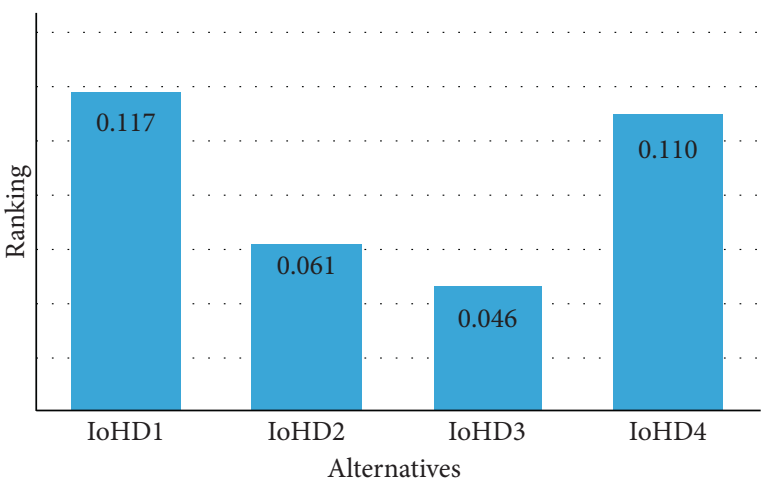

FIGURE 3: Ranking of the available alternatives (IoHDs).

From the figure, it is shown that IoHD1 is the best choice among the available alternatives. This is followed by IoHD4.

\section{Conclusions}

With the revolution in digital technology and Internet, every organization is adopting digital things to carry out their dayto-day activities. IoT is a perception used to link various devices over the Internet for increasing the production and quality of service, deliver a huge amount of data in seconds, and automate the processes of daily life. IoT implementation in the industrial sector has transformed the typical industrial setup into smart and intellectual setup. With the "smart" and "Intelligent" abilities of IoT devices such as sensors and with the collaboration of humans and computers, physical processes can be monitored and, based on the received data, optimal decisions can ultimately be taken. Industry 4.0 is increasing flexibility, mass customization, quality, and productivity. The Internet of Things associates various heterogeneous devices, and unfortunate determination of the degree of characteristics of $\mathrm{IoH}$ devices may affect the effectiveness of services. Keeping in view this issue, the proposed research is carried out with the use of decision support system and application of fuzzy analytic network process was considered for optimum determination of the degree of characteristics of $\mathrm{IoH}$ devices based on exclusive properties which would significantly upturn the efficiency of industry and its endeavors.

\section{Data Availability}

No data were used to support this study.

\section{Conflicts of Interest}

The author declares that there are no conflicts of interest regarding the publication of this paper.

\section{References}

[1] D. Zhang, C. C. Chan, and G. Y. Zhou, "Enabling industrial internet of things (IIoT) towards an emerging smart energy system," Global energy interconnection, vol. 1, no. 1, pp. 39-47, 2018.
[2] L. Urquhart and D. McAuley, "Avoiding the internet of insecure industrial things," Computer Law \& Security Report, vol. 34, no. 3, pp. 450-466, 2018.

[3] S. Vaidya, P. Ambad, and S. Bhosle, "Industry 4.0-a glimpse," Procedia manufacturing, vol. 20, pp. 233-238, 2018.

[4] M. Mahbub, "Comparative link-level analysis and performance estimation of channel models for IIoT (industrial-IoT) wireless communications," Internet of Things, vol. 12, Article ID 100315, 2020.

[5] L. Silvestri, A. Forcina, V. Introna, A. Santolamazza, and V. Cesarotti, "Maintenance transformation through industry 4.0 technologies: a systematic literature review," Computers in Industry, vol. 123, p. 103335, 2020.

[6] R. Y. Zhong, X. Xu, E. Klotz, and S. T. Newman, "Intelligent manufacturing in the context of industry 4.0: a review," Engineering, vol. 3, no. 5, pp. 616-630, 2017.

[7] L. Barreto, A. Amaral, and T. Pereira, "Industry 4.0 implications in logistics: an overview," Procedia Manufacturing, vol. 13, pp. 1245-1252, 2017.

[8] F. Civerchia, S. Bocchino, C. Salvadori, E. Rossi, L. Maggiani, and M. Petracca, "Industrial internet of things monitoring solution for advanced predictive maintenance applications," Journal of Industrial Information Integration, vol. 7, pp. 4-12, 2017.

[9] C. Cronin, A. Conway, and J. Walsh, "Flexible manufacturing systems using IIoT in the automotive sector," Procedia Manufacturing, vol. 38, pp. 1652-1659, 2019.

[10] Q. Li, Y. Yue, and Z. Wang, "Deep Robust Cramer Shoup delay optimized fully homomorphic for IIOT secured transmission in cloud computing," Computer Communications, vol. 161, pp. 10-18, 2020.

[11] A. Xenakis, A. Karageorgos, E. Lallas, A. E. Chis, and H. González-Vélez, "Towards distributed IoT/cloud based fault detection and maintenance in industrial automation," Procedia Computer Science, vol. 151, pp. 683-690, 2019.

[12] F. Zezulka, P. Marcon, Z. Bradac, J. Arm, T. Benesl, and I. Vesely, "Communication systems for industry 4.0 and the IIoT,” IFAC-PapersOnLine, vol. 51, no. 6, pp. 150-155, 2018.

[13] L. Zhou, H. Guo, and G. Deng, "A fog computing based approach to DDoS mitigation in IIoT systems," Computers \& Security, vol. 85, pp. 51-62, 2019.

[14] J. Arm, F. Zezulka, Z. Bradac et al., "Implementing industry 4.0 in discrete manufacturing: options and drawbacks," IFACPapersOnLine, vol. 51, no. 6, pp. 473-478, 2018.

[15] F. Al-Obaidy, F. Yazdani, and F. A. Mohammadi, "Intelligent testing for arduino UNO based on thermal image," Computers \& Electrical Engineering, vol. 58, pp. 88-100, 2017.

[16] S. Goudarzi, M. H. Anisi, A. H. Abdullah, J. Lloret, S. A. Soleymani, and W. H. Hassan, "A hybrid intelligent model for network selection in the industrial internet of things," Applied Soft Computing, vol. 74, pp. 529-546, 2019.

[17] M. A. Khan and K. Salah, "IoT security: review, blockchain solutions, and open challenges," Future Generation Computer Systems, vol. 82, pp. 395-411, 2018.

[18] W. Liu, G. Huang, A. Zheng, and J. Liu, "Research on the optimization of IIoT data processing latency," Computer Communications, vol. 151, pp. 290-298, 2020.

[19] X. Luo, J. Liu, D. Zhang, and X. Chang, "A large-scale web QoS prediction scheme for the industrial internet of things based on a kernel machine learning algorithm," Computer Networks, vol. 101, pp. 81-89, 2016.

[20] M. Al-Hawawreh, E. Sitnikova, and F. den Hartog, "An efficient intrusion detection model for edge system in brownfield industrial internet of things," in Proceedings of the 
3rd International Conference on Big Data and Internet of Things, pp. 83-87, Melbourn, Australia, August 2019.

[21] J. Dobaj, J. Iber, M. Krisper, and C. Kreiner, "A microservice architecture for the industrial internet-of-things," in Proceedings of the 23rd European Conference on Pattern Languages of Programs, pp. 1-15, Irsee, Germany, July 2018.

[22] M. Al-Hawawreh and E. Sitnikova, "Industrial internet of things based ransomware detection using stacked variational neural network," in Proceedings of the 3rd International Conference on Big Data and Internet of Things, pp. 126-130, Melbourn, Australia, August 2019.

[23] J. Ruh and W. Steiner, "The need for deterministic virtualization in the industrial internet of things," in Proceedings of the Workshop on Fog Computing and the IoT, pp. 26-30, Montreal, Canada, April 2019.

[24] C. Kan, H. Yang, and S. Kumara, "Parallel computing and network analytics for fast Industrial internet-of-things (IIoT) machine information processing and condition monitoring," Journal of Manufacturing Systems, vol. 46, pp. 282-293, 2018.

[25] S. Berger, O. Bürger, and M. Röglinger, "Attacks on the industrial internet of things-development of a multi-layer taxonomy," Computers \& Security, vol. 93, Article ID 101790, 2020.

[26] J. Wan, J. Li, Q. Hua, A. Celesti, and Z. Wang, "Intelligent equipment design assisted by cognitive internet of things and industrial big data," Neural Computing \& Applications, vol. 32, no. 9, pp. 4463-4472, 2020.

[27] N. Jayakumar and D. P. Joshi, "Big data \& disruptive computing platforms braced internet of things: facets \& trends," in Studies in Systems, Decision and Control, Internet of Things, Smart Computing and Technology: A Roadmap Ahead, pp. 119-150, Springer, Berlin, Germany, 2020.

[28] J. Chanchaichujit, A. Tan, F. Meng, and S. Eaimkhong, "Internet of things (IoT) and big data analytics in healthcare," in Healthcare 4.0, pp. 17-36, Springer, Berlin, Germany, 2019.

[29] E. Sisinni, A. Saifullah, S. Han, U. Jennehag, and M. Gidlund, "Industrial internet of things: challenges, opportunities, and directions," IEEE Transactions on Industrial Informatics, vol. 14, no. 11, pp. 4724-4734, 2018.

[30] H. Boyes, B. Hallaq, J. Cunningham, and T. Watson, "The industrial internet of things (IIoT): an analysis framework," Computers in Industry, vol. 101, pp. 1-12, 2018.

[31] J. H. Park, Advances in Future Internet and the Industrial Internet of Things, Multidisciplinary Digital Publishing Institute, Basel, Switzerland, 2019.

[32] Y. Chen, G. Lee, L. Shu, and N. Crespi, "Industrial internet of things-based collaborative sensing intelligence: framework and research challenges," Sensors, vol. 16, no. 2, p. 215, 2016.

[33] A. Liu, Q. Zhang, Z. Li, Y.-j. Choi, J. Li, and N. Komuro, "A green and reliable communication modeling for industrial internet of things," Computers \& Electrical Engineering, vol. 58, pp. 364-381, 2017.

[34] H. Xu, W. Yu, D. Griffith, and N. Golmie, "A survey on industrial internet of things: a cyber-physical systems perspective," IEEE Access, vol. 6, pp. 78238-78259, 2018.

[35] W. Z. Khan, M. H. Rehman, H. M. Zangoti, M. K. Afzal, N. Armi, and K. Salah, "Industrial internet of things: recent advances, enabling technologies and open challenges," Computers \& Electrical Engineering, vol. 81, Article ID 106522, 2020.

[36] H. Ezz El-Din and D. H. Manjaiah, "Internet of nano things and industrial internet of things," in Internet of Things: Novel Advances and Envisioned Applications, pp. 109-123, Springer, Berlin, Germany, 2017.
[37] H. Mouratidis and V. Diamantopoulou, "A security analysis method for industrial internet of things," IEEE Transactions on Industrial Informatics, vol. 14, no. 9, pp. 4093-4100, 2018.

[38] Q. Yan, W. Huang, X. Luo, Q. Gong, and F. R. Yu, "A multilevel DDoS mitigation framework for the industrial internet of things," IEEE Communications Magazine, vol. 56, no. 2, pp. 30-36, 2018.

[39] C. Yin, J. Xi, R. Sun, and J. Wang, "Location privacy protection based on differential privacy strategy for big data in industrial internet of things," IEEE Transactions on Industrial Informatics, vol. 14, no. 8, pp. 3628-3636, 2017.

[40] W. Chen, "Intelligent manufacturing production line data monitoring system for industrial internet of things," Computer Communications, vol. 151, pp. 31-41, 2020.

[41] N. Muthukumar, S. Srinivasan, K. Ramkumar, D. Pal, J. Vain, and S. Ramaswamy, "A model-based approach for design and verification of industrial internet of things," Future Generation Computer Systems, vol. 95, pp. 354-363, 2019.

[42] A.-H. Muna, N. Moustafa, and E. Sitnikova, "Identification of malicious activities in industrial internet of things based on deep learning models," Journal of information security and applications, vol. 41, pp. 1-11, 2018.

[43] Z. Ayağ, R. G. Özdemİr, An intelligent approach to ERP software selection through fuzzy ANP," International Journal of Production Research, vol. 45, no. 10, pp. 2169-2194, 2007. 